\title{
La amenaza del desconocimiento en la conservación de la diversidad vegetal europea: El caso de la adormidera marítima
}

\author{
J. Cambrollé ${ }^{1, *}$, S. Muñóz-Valles ${ }^{1}$, T. Luque $^{1}$, M.E. Figueroa ${ }^{1}$ \\ (1) Dpto. de Biología Vegetal y Ecología, Facultad de Biología, Universidad de Sevilla, Apartado 1095, 41080, Sevilla, España. \\ * Autor de correspondencia: J. Cambrollé [cambrolle@us.es]
}

> Recibido el 1 de mayo de 2011, aceptado el 3 de agosto de 2012.

\begin{abstract}
Cambrolle, J., Muñóz-Vallés, S., Luque, T., Figueroa, M.E. (2012). La amenaza del desconocimiento en la conservación de la diversidad vegetal europea: El caso de la adormidera marítima. Ecosistemas 21(3):110-112. Doi.: 10.7818/ECOS.2012.21-3.16

La industrialización y los cambios en el uso del territorio han originado que la flora europea esté considerada entre las más amenazadas del planeta. A pesar de los esfuerzos realizados, los datos que se manejan actualmente en relación a un gran número de especies son insuficientes para realizar una evaluación de su estado de conservación. Un ejemplo interesante lo encontramos en la "adormidera marítima" (Glaucium flavum). Aunque diferentes estudios indican que la sobreexplotación, transformación y eliminación de ecosistemas costeros durante las últimas décadas han afectado en gran medida a la conservación de esta especie, los datos que se manejan actualmente son insuficientes para realizar su evaluación a gran escala. Es necesario realizar un esfuerzo adicional para ampliar la información existente sobre el estado de conservación de ciertas especies que, a pesar de no encontrarse reconocidas como amenazadas, podrían estar experimentando importantes disminuciones en su área de distribución.
\end{abstract}

Palabras clave: Flora europea, Conservación, Glaucium flavum.

Cambrolle, J., Muñóz-Vallés, S., Luque, T., Figueroa, M.E. (2012). The threat posed by lack of knowledge in European plant diversity conservation: The case of the yellow- horned poppy. Ecosistemas 21(3):110-112. Doi.: 10.7818/ECOS.2012.21-3.16

Due to the effects of industrialization and land use change, the European flora is now considered among the most threatened worldwide. Despite all efforts, currently available data are insufficient to evaluate the conservation status of a great number of species. One interesting example is the "yellow horned poppy" (Glaucium flavum). Various studies indicate that the overexploitation, transformation and elimination of coastal ecosystems over recent decades have affected the conservation of this species to a large extent. However, the paucity of relevant data precludes a large-scale evaluation. An additional effort is therefore necessary to expand the existing information regarding the conservation status of certain species that, while not recognized as threatened, could be undergoing significant reductions in their areas of distribution.

Key words: European flora, Conservation, Glaucium flavum.

La conservación de la biodiversidad se ha convertido en un objetivo prioritario reconocido por los gobiernos nacionales, agencias estatales, comunidades locales y organizaciones científicas. La biodiversidad, junto a otros factores (como la composición de especies, el régimen de perturbaciones y las características del suelo y el clima), influye de manera significativa sobre la estabilidad, la productividad y otra serie de aspectos del funcionamiento de los ecosistemas. Además, la pérdida de biodiversidad disminuye la capacidad de los ecosistemas para proporcionar a la sociedad un suministro estable y sostenible de bienes y servicios esenciales (Tilman 2000). Por ejemplo, en la actualidad se estima que existen entre 50000 y 70000 especies vegetales utilizadas en medicina tradicional y moderna (Schippmann et al. 2006).

La biodiversidad global está disminuyendo a una velocidad sin precedentes como respuesta compleja ante los diferentes cambios inducidos por el ser humano en el ambiente global (Sala et al. 2000). En la actualidad, se estima que tan solo se ha evaluado el estado de conservación de aproximadamente un $2.7 \%$ del total de especies sobre la Tierra. La Lista Roja de la UICN (2008) incluye la evaluación de 12055 especies de plantas, de las cuales 8457 han sido clasifi- cadas como amenazadas, sin embargo, el total de especies evaluadas tan solo representa aproximadamente el $4 \%$ del total de especies vegetales conocidas en la actualidad (Nic Lughadha et al. 2005; Vié et al. 2009).

La región europea, que se extiende desde zonas de clima Mediterráneo sub-tropical hasta regiones sub-polares frías, cubre una amplia variedad de ambientes y hábitats, en los que se encuentra una gran diversidad floral. El continente europeo cuenta con aproximadamente 14000 especies de plantas vasculares (Sharrock y Jones 2011). Los principales centros de diversidad vegetal incluyen el área en torno al Mediterráneo y el Mar Negro, siendo las floras de España, Grecia, Italia y Bulgaria las más ricas en especies endémicas (Planta Europa 2002). La mayor diversidad vegetal del continente europeo se encuentra en la región mediterránea, que está considerada como uno de los 34 puntos calientes de biodiversidad global (Mittermeier et al. 2004).

La flora europea, que representa uno de los valores naturales más importantes del continente, se encuentra amenazada por diferentes factores. La continua pérdida de biodiversidad se ha convertido en uno de los principales problemas ambientales de Europa. 
Según la "Agencia Europea del Ambiente" (EEA), menos de la mitad de las especies protegidas y hábitats de Europa se encuentran en un estado favorable de conservación. Durante los dos últimos siglos, la industrialización y los cambios en el uso del territorio han originado que la flora europea esté considerada entre las más amenazadas del planeta (Sharrock y Jones 2009). Se estima que la destrucción de hábitat por la actividad humana es la principal causa de riesgo para el $83 \%$ de las especies vegetales amenazadas. En la actualidad, la flora costera europea está especialmente expuesta a las amenazas antropogénicas, debido fundamentalmente a la continua aceleración de la urbanización en las áreas costeras, consecuencia del turismo en masa y del incremento en el número de segundas viviendas (Sharrock y Jones 2009).

Los datos que se manejan actualmente en relación al estado de conservación de un gran número de especies y hábitats son simplemente insuficientes para realizar su evaluación (EEA 2006). Aunque la diversidad vegetal en Europa ha sido extensamente estudiada por una gran número de instituciones (incluyendo universidades, jardines botánicos, organizaciones gubernamentales y no gubernamentales) existe una gran cantidad de especies que, no encontrándose reconocidas como amenazadas, pueden estar experimentando importantes disminuciones en su área de distribución por degradación o destrucción de su hábitat en diferentes países (Vié et al. 2009). A lo largo de las zonas costeras de Europa existe una gran cantidad de especies vegetales que ejemplifican la citada falta de información. Un ejemplo interesante lo encontramos en la "adormidera marítima".

Glaucium flavum Crantz, comúnmente conocida como "adormidera marítima", es una planta herbácea, perteneciente a la familia Papaveraceae, que se distribuye por las zonas costeras del continente europeo y sus principales islas. Se encuentra además en el suroeste de Asia y en el noroeste de África, encontrándose naturalizada en determinadas zonas de Europa Central (Castroviejo et al. 1989). Durante las últimas décadas, ciertos estudios han detectado una importante disminución en el área de distribución de G. flavum.
Por ejemplo, Scott (1963) registró una disminución en Escocia y el norte de Inglaterra desde 1930 y en Suecia se encuentra en recesión desde los años 50 (Weimarck 1963; Fries 1971). G. flavum está clasificada como "Vulnerable" en los países Escandinavos (Directorate for Nature Management 1999) y se encuentra protegida por ley en Suecia (Hultén y Fries 1986) y Noruega (desde 2001), además de clasificada como "en peligro de extinción" en Rumanía (Bercu et al. 2006). Aunque se desconoce el estado de conservación actual de G. flavum en la mayor parte de la Península Ibérica, los datos existentes apoyan la citada disminución de su área de distribución en diferentes regiones, principalmente las localizadas en el Norte de España (Moreno Moral et al. 2001; Prieto Fernández et al. 2007). Asimismo, un estudio reciente realizado en el Sur de España pone de manifiesto una importante disminución en el número de poblaciones de G. flavum durante las últimas décadas. Para llevar a cabo dicho estudio se recopiló un amplio número de citas históricas y referencias de localizaciones correspondientes a individuos distribuidos por toda Andalucía entre los años 1929 y 2008, que fueron visitadas para comprobar la presencia de la especie en la actualidad. Resulta destacable que en más del $60 \%$ del total de localizaciones recopiladas en el estudio no se encontraron individuos vivos. Dicha información, unida a la obtenida tras la realización de una exhaustiva búsqueda de individuos en diferentes zonas potenciales para la especie, permitió realizar una aproximación sobre el estado de conservación actual de G. flavum en la región andaluza. Los resultados analizados indicaron que la principal causa de desaparición de G. flavum en el área de estudio fue la alteración o destrucción absoluta del hábitat durante las últimas décadas, y pusieron de manifiesto la existencia de importantes riesgos para las poblaciones existentes en la región (Cambrollé et al. 2011).

Al igual que otras especies de la familia Papaveraceae, G. flavum produce una serie de alcaloides que, además de tener un importante papel ecológico, poseen propiedades terapéuticas. Así, la mayor parte de la información existente sobre esta especie está relacionada con el estudio de los diferentes compuestos bioquímicos

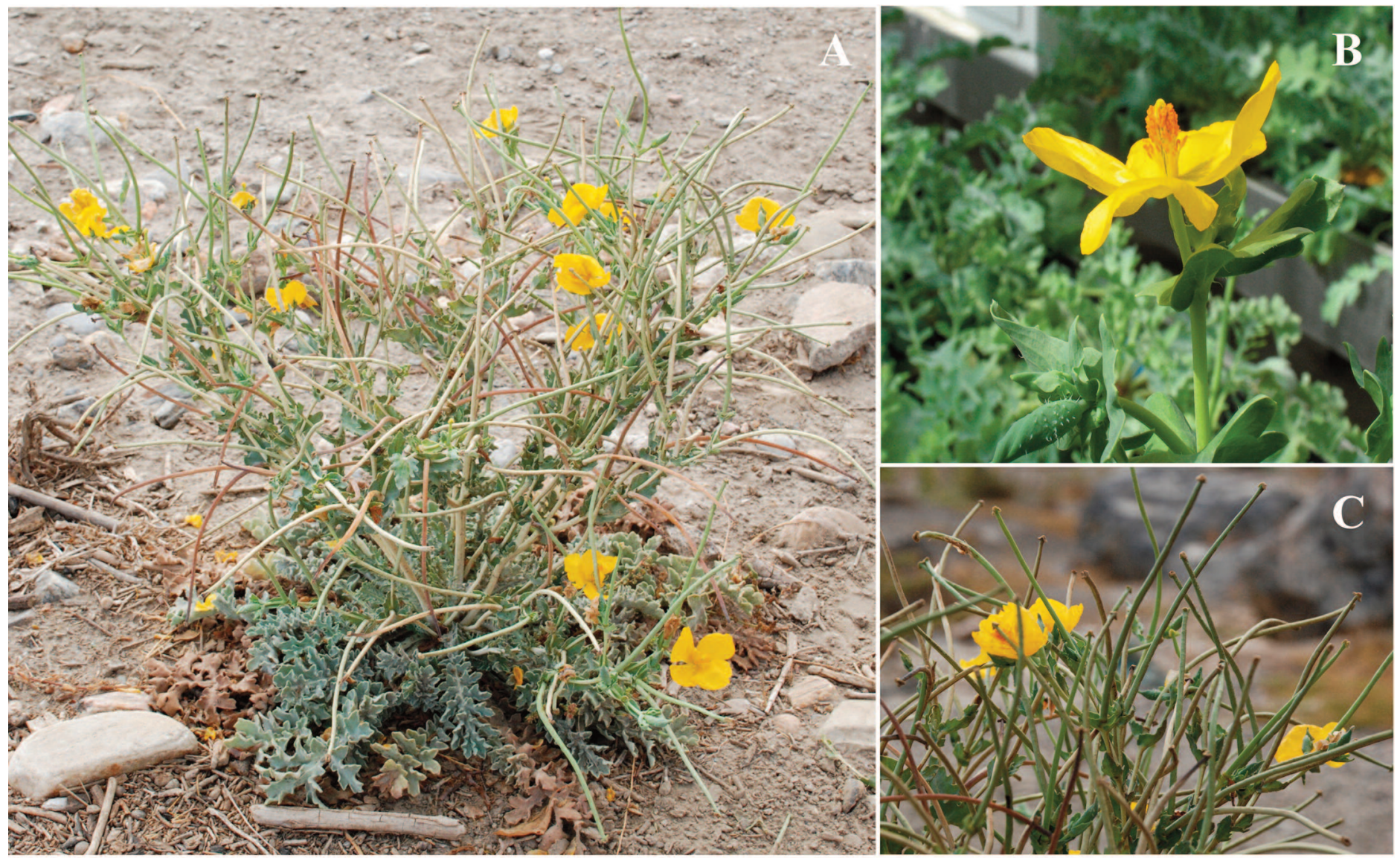

Figura 1. Glaucium flavum junto al cauce del Río Andarax, Almería (A). Detalle de la flor (B) y de los frutos (C). 
producidos por la planta, existiendo un importante vacío en cuanto al conocimiento del estado actual de conservación de la especie en la mayor parte de su área de distribución. Aunque existen indicios relevantes que indican que la destrucción del hábitat de G. flavum durante las últimas décadas ha debido afectar en gran medida a la conservación de esta especie costera, los datos que se manejan actualmente en relación a su estado de conservación son insuficientes para realizar su evaluación a gran escala.

Son muchos los proyectos en desarrollo y los esfuerzos realizados por innumerables organizaciones, instituciones y grupos para ampliar los conocimientos actuales sobre las especies vegetales del continente europeo. Inevitablemente, la limitación de recursos económicos a menudo acrecienta la tendencia a concentrar todos los esfuerzos en el estudio de las especies incluidas en la Lista Roja de Especies Amenazadas de la UICN (IUCN 2012) o en los diferentes catálogos realizados a nivel local. Sin embargo, es necesario realizar un esfuerzo adicional de coordinación, recopilación de datos y actualización de la información existente sobre el estado de conservación de ciertas especies que, como G. flavum, a pesar de no encontrarse reconocidas como amenazadas, podrían estar experimentando importantes disminuciones en su área de distribución.

\section{Referencias}

Bercu, R., Fagaras, M., Jianu, L.D. 2006. Anatomy of the endangered plant Glaucium flavum $\mathrm{Cr}$., occurring on the Romanian Black Sea litoral. En: Gafta, D. y Akeroyd, J. (eds.). Nature Conservation. Concepts and Practice, pp. 273-280, Springer-Verlag, Berlin Heidelberg. Germany.

Castroviejo, S., Laínz, M., López, G., Montserrat, P., Muñoz, F., Paiva, J., Villar, L. (eds.). A partir de 1989. Flora Ibérica. Plantas vasculares de la Península Ibérica e Islas Baleares. Real Jardín Botánico, CSIC, Madrid, España.

Cambrollé, J. 2011. Ecología, ecofisiología y estudio del estado de conservación de Glaucium flavum Crantz. Tesis Doctoral, Universidad de Sevilla, España.

Directorate for Nature Management 1999. Nasjonal rødliste for truete arter i Norge. DN raport, Ministry of Enviroment. Sweden.

European Environment Agency 2006. Progress towards halting the loss of biodiversity by 2010. EEA Report No 5/2006, EEA, Copenhagen, Denmark.Disponible en: http://www.eea.europa.eu/publications/eea_report_2006_5

Fries, H. 1971. Göteborg och Bohus läns fanerogamer och ormbunkar. Bohusläningens AB, Uddevalla, Sweden.

Hultén, E., Fries, M. 1986. Atlas of North European vascular plants north of the tropic of Cancer. Koeltz Scientific Books, Königstein, Germany.
IUCN 2012. The IUCN Red List of Threatened Species. Version 2012.2. Disponible en: http://www.iucnredlist.org.

Mittermeier, R.A., Robles Gil, P., Hoffman, M., Pilgrim, J., Brooks, T., Goettsch Mittermeier, C., Lamoreux, J., da Fonseca, G.A.B. 2004. Hotspots revisited: Earth's biologically richest and most threatened terrestrial ecoregions. Conservation International, Washington, USA.

Moreno Moral, G., Sánchez Pedraja, O., Láinz, M., Patallo, J., Aldasoro, J.J., Aedo, C. 2001. Flora de Cantabria [Bibliografía básica. Plantas amenazadas (Lista Roja de la Flora Vascular cántabra)]. Disponible en: http://grupos.unican.es/ acanto/botanica.htm\#inicio.

Nic Lughadha, E., Baillie, J., Barthlott, W., Brummitt, N.A., Cheek, M.R., Farjon, A., Govaerts, R., Hardwick, K.A., Hilton-Taylor, C., Meagher, T.R., Moat, J., Mutke, J., Paton, A.J., Pleasants, L.J., Savolainen, V., Schatz, G.E., Smith, P., Turner, I., Wyse-Jackson, P., Crane, P.R. 2005. Measuring the fate of plant diversity: towards a foundation for future monitoring and opportunities for urgent action. Philosophical Transactions of the Royal Society B 360:359-372.

Planta Europa 2002. European plant conservation strategy-saving the plants of Europe. Plantlife International, Salisbury, UK.

Prieto Fernández, A., Campos Prieto, J.A., Garmendia Altuna, J., Loidi Arregui, J., Oreja Gutiérrez, L., Patino Sánchez, S., Valencia Janices, J. 2007. Flora amenazada presente en la Región Eurosiberiana de la comunidad autónoma del País Vasco. Naturalia Cantabricae 3:79-91.

Sala, O.E., Chapin, F.S. 3rd, Armesto, J.J., Berlow, E., Bloomfield, J., Dirzo, R., Huber-Sanwald, E., Huenneke, L.F., Jackson, R.B., Kinzig, A., Leemans, R., Lodge, D.M., Mooney, H.A., Oesterheld, M., Poff, N.L., Sykes, M.T., Walker, B.H., Walker, M., Wall, D.H. 2000. Global biodiversity scenarios for the year 2100. Science 287:1770-1774.

Scott, G.A.M. 1963. Glaucium flavum Crantz. Journal of Ecology 51:733-742.

Sharrock, S., Jones, M. 2009. Conserving Europe's threatened plants: Progress towards Target 8 of the Global Strategy for Plant Conservation. Botanic Gardens Conservation International, Richmond, UK.

Sharrock, S., Jones, M. 2011. Saving Europe's threatened flora: progress towards GSPC Target 8 in Europe. Biodiversity and Conservation 20:325-333.

Schippmann, U., Leaman, D., Cunningham, A.B. 2006. Cultivation and wild collection of medicinal and aromatic plants under sustainability aspects. En: Bogers, R.J., Craker, L.E., Lange, D. (eds.). Medicinal and Aromatic Plants: Agricultural, Commercial, Ecological, Legal, Pharmacological and Social Aspects, pp. 75-95, Springer, Dordrecht. The Netherlands.

Tilman, D. 2000. Causes, consequences and ethics of biodiversity. Nature 405:208-211.

Vié, J.-C., Hilton-Taylor, C., Stuart, S.N. (eds.). 2009. Wildlife in a Changing World - An Analysis of the 2008 IUCN Red List of Threatened Species. IUCN, Gland, Swizerland.

Weimarck, H. 1963. Skånes flora. Corona AB, Lund. Sweden. 